\title{
Singularity Avoidance of Control Moment Gyros by Predicted Singularity Robustness: Ground Experiment
}

\author{
Henzeh Leeghim, Il-Hyoung Lee, Dong-Hun Lee, Hyochoong Bang, Member, IEEE, and Jong-Oh Park
}

\begin{abstract}
A steering law design for single gimbal control moment gyros (CMGs) for spacecraft attitude control is addressed. The novel approach for the new steering law has a close relevance to the well-known singularity robustness method combined with the null motion approach. The proposed predicted singularity robustness (PSR) approach ultimately leads to an optimized solution of gimbal rates with performance improvement to avoid singularity by robust gradient null vectors. To apply it to practical systems, a singularity index, so-called inner-product index, is also introduced. The null vector induced from the suggested index provides a more reliable and robust way of escaping singular states than that of the well-known condition number index. Performance of the proposed algorithm is demonstrated by using a ground experimental hardware simulator equipped with four single gimbal CMGs floating on top of an air bearing.
\end{abstract}

Index Terms-Control momentum gyros (CMGs), inner-product index, predicted singularity robustness (PSR), singularity avoidance.

\section{INTRODUCTION}

C ONTROL MOMENT GYROS (CMGs) are commonly employed to reorient large spacecraft structures by using large torque capacity. In spite of the favorable performance they should be provided with complex steering laws for practical operations, since torque direction of individual wheel continuously changes. Also, general steering laws for CMGs are usually subjected to singularity problem when torque vectors of CMGs are aligned together in the same plane or line. During the last several decades, various attempts have been made to solve the singularity problem [1]-[6]. A singularity avoidance strategy so-called the null motion approach was introduced with a series of follow-on researches [7]. The variable-speed control moment gyro (VSCMG) the null space is much larger and more flexible than the single gimbal CMG null space. It makes use of the null space to exploit redundant actuation capability of the CMG cluster to keep the gimbals angles away from singularity by adjusting wheel speeds. It represents a set of gimbal motion that produce no net control torque. A variety of analytic approaches to constructing a proper null motion have been investigated. The

\footnotetext{
Manuscript received March 03, 2008; revised September 04, 2008. Manuscript received in final form December 15, 2008. First published April 21, 2009; current version published June 24, 2009. Recommended by Associate Editor S. Kim. This work was supported in part by the STSAT-3 Program, by the Grant of MEST, and by KOMSAT- 5 development program of Korea Aerospace Research Institute (KARI).

H. Leeghim is with KAIST, Daejeon 305-701, Korea (e-mail: leeghim@ ascl. kaist.ac.kr).

I.-H. Lee, D.-H. Lee, and H. Bang are with the Division of Aerospace Engineering, KAIST, Daejeon 305-701, Korea (e-mail: ihlee@ ascl.kaist.ac.kr; dhlee@ascl.kaist.ac.kr; hcbang@ascl.kaist.ac.kr).

J.-O. Park is with the Division of Electronics Engineering, Dong-A University, Busan 604-714, Korea (e-mail: jopark@ ascl.kaist.ac.kr).

Digital Object Identifier 10.1109/TCST.2008.2011556
}

gradient method, as one of the popular null motion approaches, has received significant attention [7], [8]. For enhancement of the pseudo-inverse technique and efficient computation of the null vector, the principle of singular value decomposition (SVD) and its relationships were analyzed by Junkins and Kim [8]. This algorithm features a powerful capability to keep singular states far away by guiding gimbals to the best layout while satisfying control torque requirement. However, in a singular configuration it is not easy or impossible to compute the effective null vector, which contributes to escaping the singular state. Therefore, this approach does not always ensure escape from the singular state. As another feasible approach, the singularity robust approach was introduced in the field of robot manipulators control [9]. This technique has been extended to additional researches on the analysis of the singularity problem of CMGs [10]-[12]. With this approach, it may be possible for the CMGs to escape the singularity. This feature is different from that of the null motion approach. On the other hand, it couldn't provide a substantial way to avoid singular states or to keep them as far away as possible, and did not satisfy the control torque requirement.

An optimization approach to avoid singularity of single gimbal CMGs was proposed [13]-[15]. The singularity avoidance approach is based on an optimization technique with one-step ahead singularity cost or proper measures to be minimized. The solution derived from the approach ultimately produces optimized gimbal angular rates with improved ability to avoid singularity. This paper is an extension of [14] and [15] while demonstrating the proposed algorithm by ground experiment. If we could combine the advantages of the two approaches described in the previous paragraph, it may bring us a highly efficient singularity avoidance/escape strategy. The proposed approach leads to a solution to the question of blending the two approaches. That is, the suggested predicted singularity robustness (PSR) technique offers a robust gradient vector to escape from a singular configuration even though the CMGs are already in a singular state. Furthermore, the proposed technique can provide exact control torque simultaneously avoiding singularity. Especially, CMG steering law designers have investigated fast and reliable computational methods for computing the gradient vector, since the null motion approach is considered as a promising methodology for the singularity avoidance. A singularity index to evaluate the gradient vector is necessarily required. As a popular singularity index, non-dimensional condition number is further investigated in this paper. Furthermore, an inner-product form of singularity index candidate which is of limited use in special cases is proposed. Of course, the suggested measure is applicable to development of CMG steering laws, and offers much more reliable null vectors. 
Ground-based experiment of spacecraft attitude control offers significant benefit in terms of cost and time saving. There have been many research attempts using ground hardware simulators to test attitude control algorithms [4], [16]-[21]. In spite of limitation of achieving fine pointing accuracy due to model uncertainty and disturbances, ground experiment is still useful for quick validation of new control logic. A CMG testbed is an ideal tool to verify the new steering law at affordable cost and development time.

In this paper, a spacecraft ground experimental hardware simulator capable of three-axis attitude maneuver has been developed to demonstrate the proposed CMG steering law. For threeaxis attitude control, the simulator structure is floating in the air by an air bearing which can support up to $150 \mathrm{~kg}$ of payload. A pyramid-type CMG cluster is mounted to produce control torque. The spacecraft simulator is also isolated from the ground control computer from which attitude control commands are generated and transmitted through RF communication. With such a hardware setup, the performance of the suggested CMG steering law was demonstrated.

This paper is organized as follows. In Section I-A, one-step ahead prediction approach using the constrained optimization theory is introduced. The PSR steering law is in Section II, and then a simple index for checking singular states and reliable computation of the null vector is discussed in Section III. In Section IV, a spacecraft hardware simulator installed with a typical pyramid-type CMG cluster is introduced to demonstrate the new technique through experimental study.

\section{A. Singularity Avoidance by Optimization Approach}

A typical pyramid-type CMG cluster is illustrated in Fig. 1. This configuration has been widely investigated for the past decades. The total angular momentum vector for the four CMGs can be expressed as

$$
\boldsymbol{h}=\sum_{i=1}^{4} \boldsymbol{h}_{i}(\boldsymbol{\gamma})
$$

where $\boldsymbol{h} \in \mathcal{R}^{3}$ represents the total angular momentum vector, and $h_{i} \in \mathcal{R}^{3}$ is the $i$ th internal angular momentum vectors produced by the $i$ th CMG. The magnitude of the internal momentum vector can be assumed as $\left\|\boldsymbol{h}_{i}\right\|=1$ without loss of generality. Also, $\boldsymbol{\gamma}=\left[\gamma_{1}, \gamma_{2}, \gamma_{3}, \gamma_{4}\right]^{T}$ denotes the gimbal angle vector, $\dot{\gamma}=d \gamma / d t$ represents the gimbal angular rate vector, and $\beta$ is the skew angle of the CMGs. The total angular momentum vector of the CMG cluster can be represented by

$$
\boldsymbol{h}=\left[\begin{array}{c}
-c \beta \sin \gamma_{1}-\cos \gamma_{2}+c \beta \sin \gamma_{3}+\cos \gamma_{4} \\
\cos \gamma_{1}-c \beta \sin \gamma_{2}-\cos \gamma_{3}+c \beta \sin \gamma_{4} \\
s \beta \sin \gamma_{1}+s \beta \sin \gamma_{2}+s \beta \sin \gamma_{3}+s \beta \sin \gamma_{4}
\end{array}\right]
$$

where $\cos \beta \equiv c \beta, \sin \beta \equiv s \beta$. The time derivative of $\boldsymbol{h}$ is represented by [12]

$$
\dot{h}=A(\gamma) \dot{\gamma}=\boldsymbol{u}
$$

where $\boldsymbol{u}$ is the desired torque command vector, and $A(\gamma) \in$ $\mathcal{R}^{3 \times 4}$ is the Jacobian defined as $\partial \boldsymbol{h} / \partial \boldsymbol{\gamma}$. The control torque is usually generated from the CMG cluster by adjusting the gimbal angular rates.

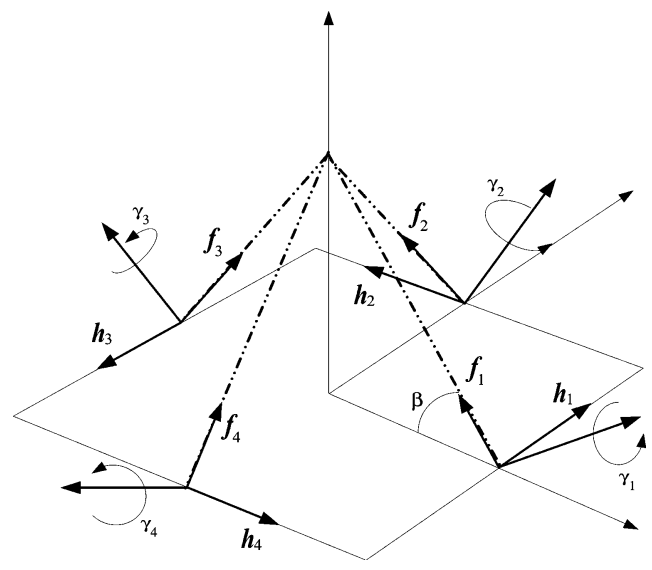

Fig. 1. Pyramid-type CMG cluster.

The optimization approach by predicting the one-step ahead singularity index is introduced in this section to help the understanding of the algorithm to be presented [14], [15]. If the rank of a range space is smaller than the number of column vectors for a given matrix, there always exists a null space. Also, different matrices have different null spaces. The Jacobian in (3) has a null space, and is always varying due to gimbal motion. It means that the null vector with the gimbal rates are always different. If we can predict the information of the matrix one-step ahead, then computation of a reliable null vector to avoid singularity may be feasible. It is the main motivation of our approach, and the performance of singularity avoidance would improve by one-step ahead prediction of the singularity measure related to the Jacobian.

A cost function is constructed such that the singularity avoidance objective is well taken into account. Since our primary goal is to avoid singularity, construction of a steering law by predicting the singular state would be a reasonable path to achieving the purpose. In other words, a singularity index is explicitly included in the cost function. To formulate the optimization problem, the cost function to be minimized with a singularity measure can be selected as [14], [15]

$$
\min _{\dot{\gamma}}\left[v V(\boldsymbol{\gamma}+\dot{\gamma} \Delta t)+\frac{1}{2} \dot{\gamma}^{T} W \dot{\gamma}\right]
$$

with a torque command requirement

$$
A(\gamma) \dot{\gamma}=u
$$

where $\boldsymbol{u} \in \mathcal{R}^{3}$ denotes the desired torque command vector, whereas $\boldsymbol{\gamma} \in \mathcal{R}^{n}$ represents the gimbal angle vector of the cluster of $n$ single gimbal CMG. The cost function $V(\boldsymbol{\gamma}+\dot{\boldsymbol{\gamma}} \Delta t)$ represents the singularity measure predicted one-step $(\Delta t)$ ahead, which should be minimized with respect to the gimbal angular rates. The scalar $v \in \mathcal{R}$ and the matrix $W \in \mathcal{R}^{n \times n}$ are the design parameter and a weighting matrix for the singularity cost and the energy cost, respectively.

A minimum-norm solution minimizing the cost function subjected to (5) can be derived by introducing Lagrange multiplier vector $(\boldsymbol{\lambda})$ such that

$$
L=v V(\dot{\gamma})+\frac{1}{2} \dot{\gamma}^{T} W \dot{\gamma}+\lambda^{T}\{A(\boldsymbol{\gamma}) \dot{\gamma}-\boldsymbol{u}\}
$$


An optimality condition is expressed as [25]

$$
\frac{\partial L}{\partial \dot{\gamma}}=v \frac{\partial V}{\partial \dot{\gamma}}+\dot{\gamma}^{T} W+\boldsymbol{\lambda}^{T} A=0
$$

It is generally difficult to obtain a closed-form solution since the singularity cost is a nonlinear function. A feasible approach is linearization of the cost function about the given gimbal angle vectors. By introducing Taylor series expansion, the singularity cost can then be expressed as

$$
\begin{aligned}
V(\boldsymbol{\gamma}+\dot{\boldsymbol{\gamma}} \Delta t)=V(\boldsymbol{\gamma})+V^{\prime}(\boldsymbol{\gamma})^{T} & \dot{\boldsymbol{\gamma}} \Delta t \\
& +\frac{1}{2} \dot{\boldsymbol{\gamma}}^{T} \Delta t V^{\prime \prime}(\boldsymbol{\gamma}) \dot{\boldsymbol{\gamma}} \Delta t+\cdots
\end{aligned}
$$

where

$$
\begin{aligned}
V^{\prime}(\gamma) & =\left[\begin{array}{lll}
\frac{\partial V(\gamma)}{\partial \gamma_{1}} & \ldots & \frac{\partial V(\gamma)}{\partial \gamma_{n}}
\end{array}\right]^{T} \in \mathcal{R}^{n} \\
V^{\prime \prime}(\gamma) & =\left[\begin{array}{ccc}
\frac{\partial^{2} V(\gamma)}{\partial \gamma_{1}^{2}} & \cdots & \frac{\partial^{2} V(\gamma)}{\partial \gamma_{n} \partial \gamma_{1}} \\
\vdots & \ddots & \vdots \\
\frac{\partial^{2} \dot{V}(\gamma)}{\partial \gamma_{1} \partial \gamma_{n}} & \cdots & \frac{\partial^{2} \dot{V}(\gamma)}{\partial \gamma_{n}^{2}}
\end{array}\right] \in \mathcal{R}^{n \times n}
\end{aligned}
$$

The partial derivative of the cost function with respect to $\dot{\gamma}$ can be written as

$$
\frac{\partial V}{\partial \dot{\boldsymbol{\gamma}}}=\Delta t V^{\prime}(\boldsymbol{\gamma})^{T}+\Delta t^{2} \dot{\boldsymbol{\gamma}}^{T} V^{\prime \prime}(\boldsymbol{\gamma})+\cdots
$$

By eliminating higher order terms, the optimality condition in (7) approximately yields an optimized solution:

$$
\frac{\partial L}{\partial \dot{\boldsymbol{\gamma}}} \approx \dot{\boldsymbol{\gamma}}^{T}(\bar{H}+W)+\boldsymbol{g}^{T}+\boldsymbol{\lambda}^{T} A=0
$$

where a Hessian $(\bar{H})$ and a gradient vector $(\boldsymbol{g})$ are defined as

$$
\bar{H}(\boldsymbol{\gamma}) \equiv \Delta t V^{\prime \prime}(\boldsymbol{\gamma}), \boldsymbol{g}(\boldsymbol{\gamma}) \equiv V^{\prime}(\boldsymbol{\gamma})
$$

and the weighting parameter $v$ is selected here as $1 / \Delta t$. Then, the optimal gimbal rate vector $\dot{\gamma}$ satisfies

$$
\dot{\boldsymbol{\gamma}}=-H^{-1}\left(A^{T} \boldsymbol{\lambda}+\boldsymbol{g}\right)
$$

where the modified Hessian $(H=\bar{H}+W)$ is newly introduced. By inserting (12) into (5), one can obtain the Lagrange multiplier vector as

$$
\lambda=-\left(A H^{-1} A^{T}\right)^{-1} \boldsymbol{u}-\left(A H^{-1} A^{T}\right)^{-1} A H^{-1} \boldsymbol{g}
$$

As a consequence, the gimbal angular rate vector $\dot{\gamma}$ is expressed as

$$
\begin{aligned}
\dot{\boldsymbol{\gamma}}=H^{-1} A^{T} & \left(A H^{-1} A^{T}\right)^{-1} \boldsymbol{u} \\
& +\left[H^{-1} A^{T}\left(A H^{-1} A^{T}\right)^{-1} A H^{-1}-H^{-1}\right] \boldsymbol{g}
\end{aligned}
$$

Note that the Hessian derived from the partial derivatives of the singularity cost may become singular since it also consists of varying gimbal vectors. From the result in (14), the direct cause of the singularity is due to the inverse of the modified Hessian $(H)$. Such a possibility can be eliminated by adjusting the weighting matrix and applying a general CMG property. Let us assume that the Hessian is a bounded linear operator. Therefore, the matrix norm of the Hessian is bounded by

$$
\|\bar{H}\|<\Delta t \nu
$$

where $\nu$ is a positive real parameter. The following inverse theorem is helpful to prove the existence of the inverse.

Inverse Theorem: Let $X$ be a linear operator, if $\|X\|<1$, then $(I-X)^{-1}$ exists as a bounded linear operator [26]. It can be readily proven by selecting the weighting matrix $W=\alpha I$ so that the modified Hessian never reaches a singular state. The modified Hessian can be reconfigured as

$$
H=\alpha\left(I+\frac{\bar{H}}{\alpha}\right)
$$

Let $-\bar{H} / \alpha$ be set to $X$. By an appropriate selection of the design parameters, the norm of $X$ can be made smaller than unity because of the boundedness of the Hessian. By the above inverse theorem, there exists a linear operator $(I+\bar{H} / \alpha)^{-1}$ as the inverse of the modified Hessian. Accordingly, it always guarantees existence of the inverse of $H$, if the weighting parameter satisfies $\alpha \geq \Delta t \nu$. Namely, if $\alpha$ and $\nu$ are fixed, then the time interval, $\Delta t$, must be smaller than $\alpha / \nu$.

\section{PRedicted Singularity Robustness (PSR)}

The minimization problem in the previous section satisfies the torque command constraint exactly. However, the well-known singularity robustness approach does not satisfy the constraint under the process of singularity avoidance in some cases, because the singularity problem is solved by adding a small variation to the steering law. The approach has widely come into focus due to the favorable feature of successful escape from singular states. The generalized singularity robustness(GSR) approach also ensures escape from any internal singularities for a non-zero constant torque input [10]. However, it may not be clear whether the singularity robustness approach ensures the best way to escape from a singular configuration. This technique allows us to avoid just current singular state.

As elaborated in the introduction part, if the inherent advantages of the PSR and null motion approaches are combined together, it may produce highly efficient performance to avoid/escape the singularity of CMGs. Thus, the strategy proposed in this section naturally pushes the singular states as far away as possible, and establishes the most desirable way to escape singular configurations by help of guiders, such as possible robust null vectors in a singular layout. Therefore, the method presented naturally gives a solution to the question of blending the two approaches. The key idea of the technique is to allow a small torque error to effectively escape from singular states by support of null vectors. The method to improving the performance of the singularity robustness approach can be constructed by properly collocating with the one-step ahead singularity prediction technique described in the previous section. To adopt the idea, the torque requirement in (5) is slightly modified by adding a torque 
error vector. The error vector here is another state to be minimized. An optimization problem can be formulated by a cost function defined as

$$
\min _{\dot{\gamma}, \delta u}\left\{\frac{1}{2} \dot{\boldsymbol{\gamma}}^{T} W \dot{\boldsymbol{\gamma}}+\delta \boldsymbol{u}^{T} R^{-1} \delta \boldsymbol{u}+v V(\boldsymbol{\gamma}+\dot{\boldsymbol{\gamma}} \Delta t)\right\}
$$

with a torque command requirement

$$
A(\boldsymbol{\gamma}) \dot{\gamma}=\boldsymbol{u}+\delta \boldsymbol{u}
$$

where $\delta \boldsymbol{u}$ denotes the torque error vector to be minimized, and $R^{-1}$ is a positive definite weighting matrix.

Assume that the weighting matrix of the torque error vector is set to infinity. It means that no torque error is allowed. The result would be identical to the method in (14). For the improved escape performance, the torque error should be inevitably created in accordance with the weighting matrix, $R^{-1}$. From the optimization method, the predicted singularity robustness(PSR) steering law is readily obtained as

$$
\begin{aligned}
\dot{\boldsymbol{\gamma}}=H^{-1} A^{T}\left(A H^{-1} A^{T}+R\right)^{-1} \boldsymbol{u} & \\
+ & {\left[H^{-1} A^{T}\left(A H^{-1} A^{T}+R\right)^{-1} A H^{-1}-H^{-1}\right] \boldsymbol{g} . }
\end{aligned}
$$

The steering law consists of the singularity robustness term as well as the gradient term. The second term enhances the performance of singularity avoidance by the one-step ahead predicted null vector with robustness due to $R$. In other words, the weighting matrix contributes more flexibility for the gradient calculation. Obviously, the flexibility would ultimately cause control torque error. However, the modified form could possibly be used to develop various versions of the steering law.

Selection of $R$ is crucial from the aspect of both torque error minimization and singularity avoidance. At first, to understand how the weighting matrix of $R$ affects the singular values of the Jacobian $(A)$, the definition of singular value decomposition (SVD) is briefly introduced.

Generally, every $m \times n$ matrix $(A)$ with $\operatorname{rank}(A) \leq m$ can be expressed in the form

$$
A=X \Sigma Y^{T}
$$

with $X^{T} X=X X^{T}=I_{m}, Y^{T} Y=Y Y^{T}=I_{n}$, where the matrices are defined as $X \equiv\left[\boldsymbol{x}_{1}, \boldsymbol{x}_{2}, \ldots, \boldsymbol{x}_{m}\right], Y \equiv$ $\left[\boldsymbol{y}_{1}, \boldsymbol{y}_{2}, \ldots, \boldsymbol{y}_{n}\right]$, respectively, and $\Sigma$ denotes the singular value matrix defined as [11]

$$
\Sigma=\left[\begin{array}{cccccc}
\sigma_{1} & 0 & \cdots & 0 & \cdots & 0 \\
0 & \ddots & \cdots & 0 & \cdots & 0 \\
0 & 0 & \sigma_{m} & 0 & \cdots & 0
\end{array}\right] \in \mathcal{R}^{m \times n} .
$$

In the previous equation, $\sigma_{i}$ represent singular values. It is assumed that they are arranged such that $\sigma_{1} \geq \sigma_{2} \cdots \geq \sigma_{m} \geq 0$.

By using SVD, the term in the steering law in (19) can be transformed into

$$
A^{T}\left(A A^{T}+R\right)^{-1}=Y \Sigma^{T}\left(\Sigma \Sigma^{T}+\Xi\right)^{-1} X^{T}
$$

where the weighting matrix $R$ is defined as

$$
R=X \Xi X^{T}
$$

the matrix $\Xi$ denotes a positive semi-definite weighting matrix, and $H$ is assumed to be an identity matrix for simplification without loss of generality. Note that the singular values $\sigma_{1}$ and $\sigma_{2}$ of a pyramid-type CMG cluster are always positive. As a singular configuration approaches, the third singular value of the Jacobian always tends to zero. To prevent the third element from becoming zero, the weighting matrix can be selected as

$$
\Xi=\operatorname{diag}\left[\begin{array}{lll}
0 & 0 & \alpha
\end{array}\right]
$$

where $\alpha$ is a small nonnegative value. Consequently, the existence of the inverse term in (22) is always guaranteed by the selected weighting matrix such that the only inverse term of the steering law can be

$$
\left(\Sigma \Sigma^{T}+\Xi\right)^{-1}=\operatorname{diag}\left[\begin{array}{lll}
\frac{1}{\sigma_{1}^{2}} & \frac{1}{\sigma_{2}^{2}} & \frac{1}{\sigma_{3}^{2}+\alpha}
\end{array}\right] .
$$

If $\alpha$ is zero with $\sigma_{3} \neq 0$ such that $R=0$ due to $\Xi=0$, then the steering law can satisfy the control torque requirement exactly without any torque error. A proper adaptation law of $\alpha$ for satisfaction of the torque command requirement and singularity avoidance is desired. One of the possible choices, for example, is [22]

$$
\alpha=\alpha_{0} \exp \left(-\alpha_{1} \sigma_{3}^{2}\right)
$$

where $\alpha_{0}$ and $\alpha_{1}$ are positive design parameters. The existence of the inverse guaranteed by the weighting matrix makes the approach more robust. Furthermore, the robust gradient vector also adds more flexibility to avid a singular configuration.

\section{SingULARITY INDEX}

The PSR method described so far requires a singularity index to adequately describe singularity condition of CMGs. In this section, two singularity indices are introduced. First, non-dimensional condition number, being used widely, is introduced. Next, an inner-product based singularity index which is shown to be reliable and robust is proposed.

Non-Dimensional Condition Number: As an index to measure the singular state of the Jacobian, condition number or non-dimensional condition number defined as

$$
V=\frac{\sigma_{1}}{\sigma_{3}}
$$

has been widely employed. A useful algorithm to quickly compute the gradient vector was introduced [8]. The partial derivatives of $V$ with respect to the gimbal angles are written as

$$
g_{i}=\frac{\partial V}{\partial \gamma_{i}}=\frac{1}{\sigma_{3}} \frac{\partial \sigma_{1}}{\partial \gamma_{i}}-\frac{\sigma_{1}}{\sigma_{3}^{2}} \frac{\partial \sigma_{3}}{\partial \gamma_{i}} .
$$


TABLE I

SPEC. OF THE SPACECRAFT SimUlatoR

\begin{aligned} \hline \hline Parts & Features \\ \hline Spacecraft structure & Moment of inertia : diag[24.3, 24.3, 32.7] $\mathrm{kg} \cdot \mathrm{m}^{2} \\ &$ Weight : $82.0 \mathrm{~kg} \\$ Flying wheel & Moment of inertia : diag[ $0.0032,-,-] \mathrm{kg} \cdot \mathrm{m}^{2} \\$ IMU(Inertial Measurement Unit) & max. angular rate : $\pm 100.0^{\circ} / \mathrm{sec} \\ &$ Rate resolution : $<0.05^{\circ} / \mathrm{sec} \\$ Onboard Flight Computer & $\mathrm{CPU}:$ Via 400MHz \\ & OS : $\mu C$ OS-II (Real-time OS) \\ Gimbal motor & Max. angular speed : 20000.0rpm \\ & Limited max. angular speed : 10.0rpm \\ Wheel motor & Max. angular speed : 20000rpm \\ & Controlled angular speed : 3500.0rpm \\ \hline \hline & \end{aligned}

By using (20), the partial derivatives of the singular values with respect to gimbal angles are given by

$$
\frac{\partial \sigma_{k}}{\partial \gamma_{i}}=\boldsymbol{x}_{k}^{T} \frac{\partial A}{\partial \gamma_{i}} \boldsymbol{y}_{k}
$$

The relationship between the Jacobian and the derivative of $\boldsymbol{h}$ in (1) with respect to the gimbal angle vector is represented by

$$
A=\frac{\partial \boldsymbol{h}}{\partial \boldsymbol{\gamma}} \equiv\left[\begin{array}{llll}
\boldsymbol{f}_{1} & \boldsymbol{f}_{2} & \boldsymbol{f}_{3} & \boldsymbol{f}_{4}
\end{array}\right] \in \mathcal{R}^{3 \times 4} .
$$

Note that $h_{i}$ are periodic functions with an interval of $2 \pi$ such that [23]

$$
\frac{\partial^{2} \boldsymbol{h}_{i}}{\partial \gamma_{i}^{2}}=\frac{\partial \boldsymbol{f}_{i}}{\partial \gamma_{i}}=-\boldsymbol{h}_{i}
$$

By applying the previous properties, (29) can be transformed into a simple form

$$
\frac{\partial \sigma_{k}}{\partial \gamma_{i}}=-y_{i k} \boldsymbol{x}_{k}^{T} \boldsymbol{h}_{i}
$$

The Hessian is needed in implementing the proposed steering law. Using (28), the partial derivatives of the gradient vector with respect to the gimbal angles can be obtained by

$$
\begin{aligned}
\frac{\partial^{2} V}{\partial \gamma_{i} \partial \gamma_{j}}= & \frac{1}{\sigma_{3}} \frac{\partial^{2} \sigma_{1}}{\partial \gamma_{i} \partial \gamma_{j}} \\
& -\frac{1}{\sigma_{3}^{2}}\left(\frac{\partial \sigma_{1}}{\partial \gamma_{i}} \frac{\partial \sigma_{3}}{\partial \gamma_{j}}+\frac{\partial \sigma_{3}}{\partial \gamma_{i}} \frac{\partial \sigma_{1}}{\partial \gamma_{j}}+\sigma_{1} \frac{\partial^{2} \sigma_{3}}{\partial \gamma_{i} \partial \gamma_{j}}\right) \\
& +\frac{2 \sigma_{1}}{\sigma_{3}^{3}} \frac{\partial^{2} \sigma_{3}}{\partial \gamma_{i} \partial \gamma_{j}} .
\end{aligned}
$$

Note that from (32) one can find a property stated as

$$
\frac{\partial^{2} \sigma_{k}}{\partial \gamma_{i} \partial \gamma_{j}}= \begin{cases}-y_{i k} \boldsymbol{x}_{k}^{T} \boldsymbol{f}_{i} & \text { if } i=j \\ 0 & \text { if } i \neq j\end{cases}
$$

By inserting the previous property into (33), the Hessian of $A$ can be readily obtained.

From (28), (33), one can find that there is one drawback of the gradient and Hessian derived from the non-dimensional condi-

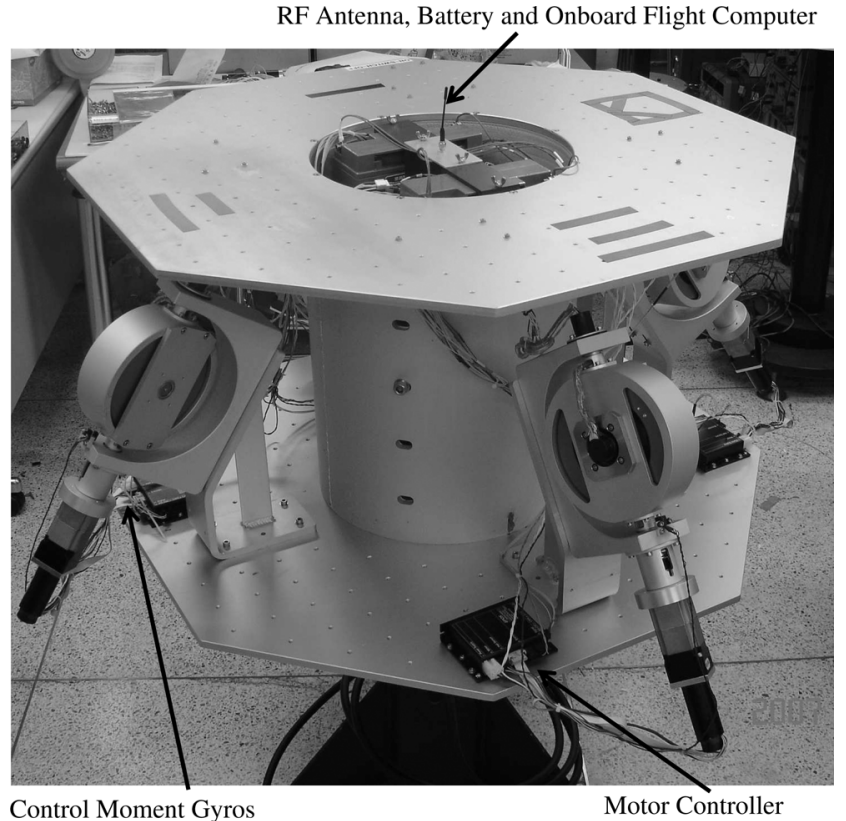

Fig. 2. Ground experimental hardware for CMG tests.

tion number. Assuming that $\sigma_{3} \approx 0$, one can see the denominator of the gradient and Hessian approach near zero. Finally, they force the gimbals to produce very large angular rates and cause numerical computation to become unstable. To overcome such a problematic scenario, a new singularity index is introduced for robustness and reliability.

Inner-Product Index: A simple singularity index is introduced to compute the gradient and Hessian with reliability. Note that if all the inner-products of two different column vectors of a given matrix are zero, then the column vectors are orthogonal to each other. This means that a matrix with all nonzero length columns, with all dot products of pairs of columns being zero, is maximal rank. With this idea, the so-called inner-product index is defined as

$$
V=\frac{1}{2} \sum_{i=j=1, i \neq j}^{4}\left\langle\boldsymbol{f}_{i}, \boldsymbol{f}_{j}\right\rangle^{2}
$$

where $\langle$,$\rangle denotes the inner-product operator. When the pro-$ posed index is zero or minimum, one can conclude that the matrix is full rank or at least maximum rank. Thus, the suggested index could be taken as a possible singularity index. Note that the norm of the column vectors $\boldsymbol{f}_{i}$ should be all nonzero values. If not, the index cannot be replaced with the singularity index. Fortunately, the column vectors of Jacobian in (30) are all unit vectors. If a column vector of the Jacobian is assumed to be a zero vector, it implies that the wheel with zero momentum does not function as a CMG. As a consequence, the given index can be used to quantify singularity level for the Jacobian.

First, the gradient vector can be readily obtained as

$$
\boldsymbol{g}=\sum_{i=j=1, i \neq j}^{4}\left(\boldsymbol{f}_{i}^{T} \boldsymbol{f}_{j}\right) \frac{\partial\left(\boldsymbol{f}_{i}^{T} \boldsymbol{f}_{j}\right)}{\partial \boldsymbol{\gamma}}
$$




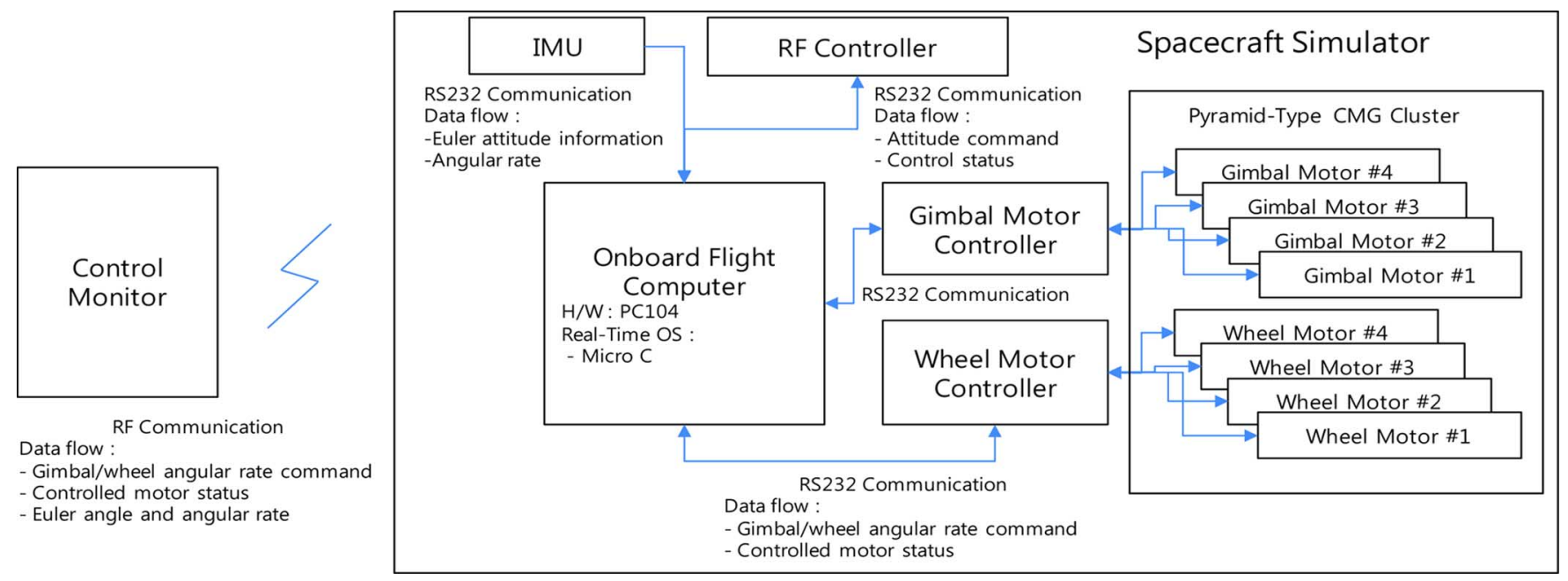

Fig. 3. Overall system block diagram for the real-time controller.

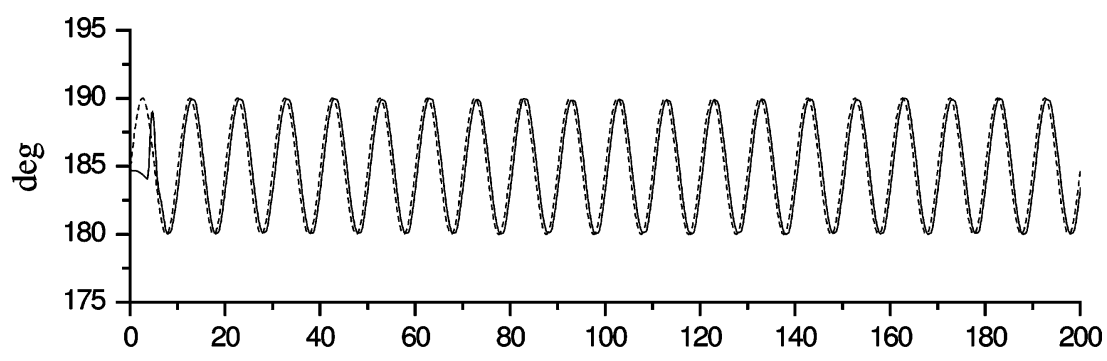

a)

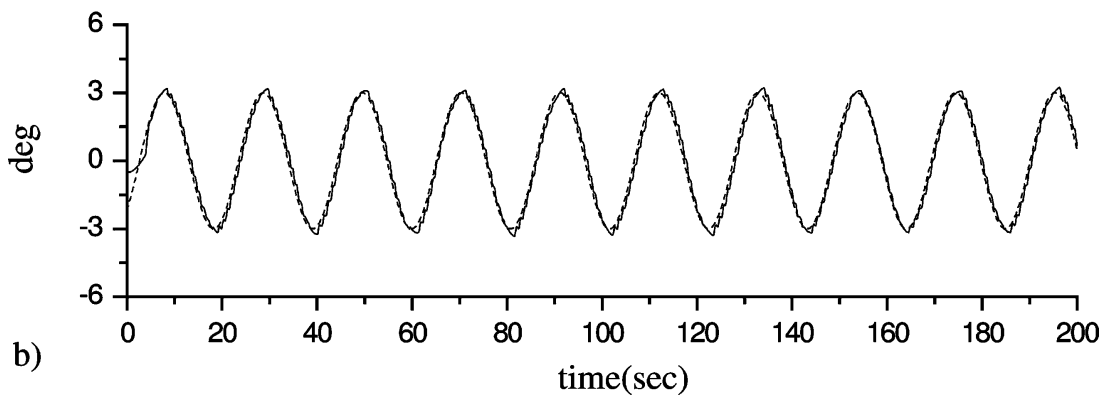

Fig. 4. History of pitch/yaw commands and experimental results. (a) Yaw angle. (b) Pitch angle.

By using (31), the partial derivative of the inner-product with respect to the gimbal angles is represented by

$$
\frac{\partial\left(\boldsymbol{f}_{i}^{T} \boldsymbol{f}_{j}\right)}{\partial \gamma_{i}}=-\boldsymbol{h}_{i}^{T} \boldsymbol{f}_{j}
$$

The $i$ th element of the gradient vector is arranged in a compact form such that

$$
g_{i}=-\sum_{j=1, j \neq i}^{4}\left(\boldsymbol{f}_{i}^{T} \boldsymbol{f}_{j}\right)\left(\boldsymbol{h}_{i}^{T} \boldsymbol{f}_{j}\right) .
$$

Next, elements of the Hessian, partial derivatives of the gradient vector with respect to the gimbal angles, are also given by

$$
\begin{aligned}
\frac{\partial^{2} V}{\partial \gamma_{i}^{2}} & =\sum_{j=1, j \neq i}^{4}\left(\boldsymbol{h}_{i}^{T} \boldsymbol{f}_{j}\right)^{2}-\left(\boldsymbol{f}_{i}^{T} \boldsymbol{f}_{j}\right)^{2} \\
\frac{\partial^{2} V}{\partial \gamma_{i} \partial \gamma_{j}} & =\left(\boldsymbol{f}_{i}^{T} \boldsymbol{h}_{j}\right)\left(\boldsymbol{h}_{i}^{T} \boldsymbol{f}_{j}\right)+\left(\boldsymbol{f}_{i}^{T} \boldsymbol{f}_{j}\right)\left(\boldsymbol{h}_{i}^{T} \boldsymbol{h}_{j}\right) .
\end{aligned}
$$

Only the angular momentum $\left(\boldsymbol{h}_{i}\right)$ and torque vector $\left(\boldsymbol{f}_{i}\right)$ are involved with evaluating the gradient vector and Hessian. They can be quickly evaluated by using the proposed inner-product form of singularity index. From the results, it is evident that the gimbal rates created by the suggested index work as a more reliable measure than the non-dimensional condition number.

\section{GRound TESTS}

For three-axis attitude control demonstration, a spacecraft simulator structure designed is floating in the air by an air bearing which can support up to $150 \mathrm{~kg}$ as illustrated in Fig. 2 . This simulator hardware system consists of a supporting structure, eight motors for running gimbals and wheels, respectively, eight motor controllers, an inertial measurement unit (IMU) for sensing three-axis attitudes and angular rates of the octagon-shaped structure. In addition, a RF controller for communication between the simulator and a ground computer, and an on-board computer for the attitude control using CMGs 


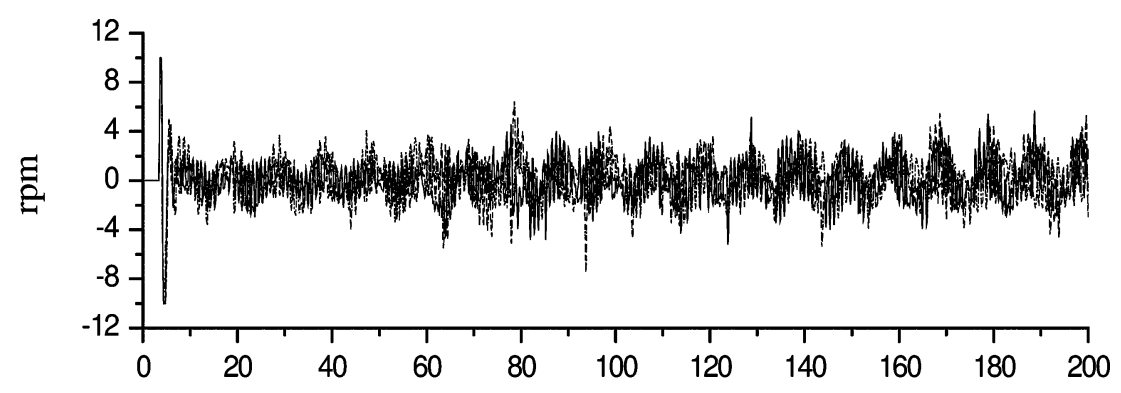

a)

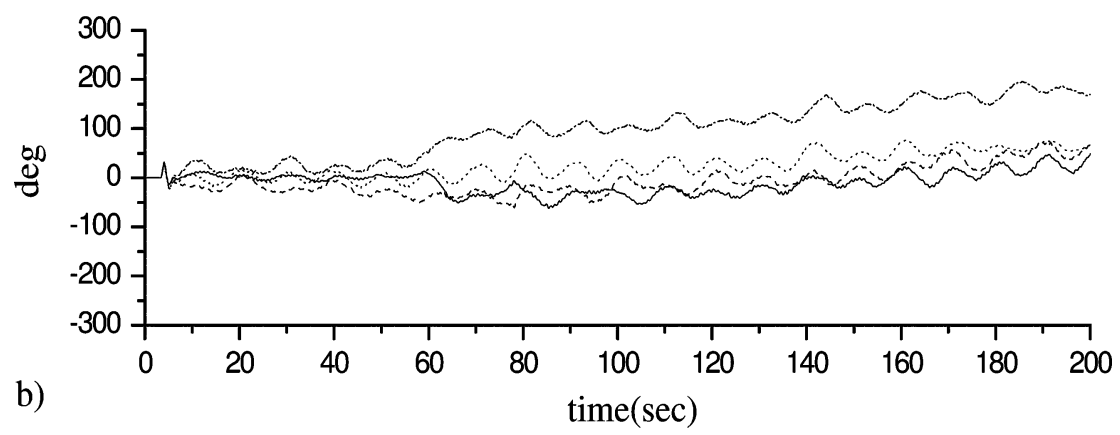

Fig. 5. Experimental response of the gimbal angular rates and angles. (a) Angular rate of gimbals. (b) Angle of gimbals.

are installed. The roll and pitch angle are limited to 6 degrees, whereas the yaw angle about the vertical axis is unlimited.

Overall specifications of the simulated spacecraft hardware including a pyramid-type CMG cluster and several hardware systems are listed in Table I. The moment of inertias of the spacecraft structure and wheels are roughly estimated by CATIA CAD design tool. Thus exact values of them are unknown and the best estimation of the parameters are out of scope in this paper. Attitude control computer is designed using PC-104 hardware single board computer system. The control computer features several interfaces ports to communicate with the gimbal/wheel motor controllers, the IMU, and the RF controller. For real-time attitude control, $\mu \mathrm{C}$ OS-II operating system is selected. The attitude control update frequency is 20 $\mathrm{Hz}$ such that the speed commands of the gimbal motors are issued at the same frequency.

The overall system block diagram of the onboard attitude controller using CMGs is also illustrated in Fig. 3. Each part can communicate with other subsystems through serial interface (RS232C). Data between the ground control computer and the spacecraft simulator are transmitted by RF communication interface. With on-board power system, this configuration allows us to operate the spacecraft simulator without much external disturbance sources due to messy line connections.

To demonstrate the proposed PSR approach and the innerproduct index, experimental study is performed. The nominal angular speed of the flying wheels is set to $3500 \mathrm{r} / \mathrm{min}$. Sinusoidal form of attitude commands are generated from the control computer. Experimental results of the attitude control using the proposed approach are plotted in Fig. 4. The attitude control results by the proposed algorithm show perfect match with the commands. The next figure shows the CMG's responses of the above attitude control experiment. In this experiment, the max-

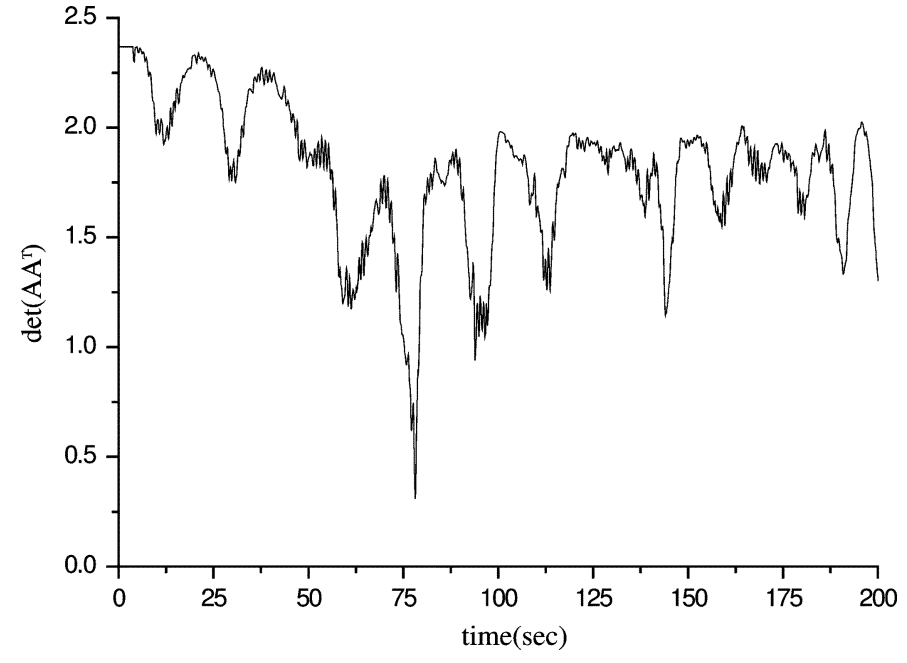

Fig. 6. Singularity index of the CMG cluster.

imum gimbal rate is limited to $10.0 \mathrm{rpm}$ due to hardware specifications. For production of desired control torque, response of the gimbal rates is plotted in Fig. 5(a). The steering law based on the PSR technique always offers allowable angular speed of gimbals such that the required control torque is also generated in smooth profiles. From the gimbal angles illustrated in Fig. 5(b), one can see that the proposed steering law is claimed to be reliable and flexible. The performance of the CMG steering law can be readily proven by analyzing the singularity index. The last plot in Fig. 6 shows determinant of the Jacobian as a singularity index. If the index reaches zero, it implies singularity. Note that there can be various sources to make it difficult to control the attitude of the simulator. One of the major sources is gravity effect by which the unwanted disturbance torque is 
induced due to mass unbalance of the simulator. These effects make CMGs easily fall into singularity during operation. Nevertheless, the PSR technique displayed in Fig. 6 always provides a substantial performance to keep the singularity away from the current layout with the help of the robust null vector obtained from the inner-product index. It is the reason why the attitude control torque is always generated in a stable fashion without any torque error.

\section{CONCLUSION}

The predicted singularity robustness (PSR) technique for CMGs steering law is synthesized using the singularity robustness technique combined with the one-step ahead singularity prediction technique. The development of a novel steering law for control moment gyros provides additional insight for singularity avoidance. One can conclude that the approach has some favorable features to avoid singularity efficiently. Some unique cost functions lead to different results to avoid singularity of CMGs. It means that choice of a new cost function results in an effective singularity avoidance algorithm. The inner-product index for measuring the singularity seems to offer reliability and consistency in evaluation of the gradient vector and Hessian for deriving the CMG steering laws. Ground experimental demonstration further strengthens the potential capability of the proposed algorithm. Despite many technical limitations in the ground experimental study, meaningful results were obtained highlighting the value of experiment.

\section{REFERENCES}

[1] D. E. Cornick, "Singularity avoidance conrol laws for single gimbal control moment gyros," AIAA Paper 79-1698, Aug. 1979.

[2] S. R. Vadali, H. Oh, and S. Walker, "Preferred gimbal angles for singlegimbal conrol moment gyroscopes," J. Guid., Control, Dyn., vol. 13, no. 6, pp. 1090-1095, 1990.

[3] H. Lee, S. Cho, and H. Bang, "Attitude control of agile spacecraft using momentum exchange devices," KSAS Int. J., vol. 7, no. 2, pp. 14-25, 2006.

[4] H. Kurokawa, "Constrained steering law for pyramid-type control moment gyros and ground Tests," J. Guid., Control, Dyn., vol. 20, no. 3, pp. 445-449, 1997.

[5] H. Oh and S. R. Vadali, "Feedback control and steering laws for spacecraft using single gimbal control moment gyros," J. Astronautical Sci., vol. 39, no. 2, pp. 183-203, 1994.

[6] N. S. Bedrossian, J. Paradiso, E. V. Bergmann, and D. Rowell, "Steering law design for redundant single-gimbal control moment gyroscopes," J. Guid., Control, Dyn., vol. 13, no. 6, pp. 1083-1089, 1990.
[7] H. Schaub and J. L. Junkins, "Singularity avoidance using null motion and variable-speed control moment gyros," J. Guid., Control, Dyn., vol. 23, no. 1, pp. 11-16, 2000.

[8] J. L. Junkins and Y. Kim, Introduction to Dynamics and Control of Flexible Structures. Washington, DC: AIAA, 1993, pp. 9-64.

[9] Y. Nakamura and H. Hanafusa, "Inverse kinematic solutions with singularity robustness for robot manipulator control," J. Dyn. Syst., Meas., Control, vol. 108, pp. 163-171, Sep. 1986.

[10] B. Wie, D. Bailey, and C. Heiberg, "Singularity robust steering logic for redundant single-gimbal control moment gyros," J. Guid., Control, Dyn., vol. 24, no. 5, pp. 865-872, 2001.

[11] K. A. Ford and C. D. Hall, "Singular direction avoidance steering for control-moment gyros," J. Guid., Control, Dyn., vol. 23, no. 4, pp. 648-656, 2000

[12] B. Wie, Space Vehicle Dynamics and Control. Washington, DC: AIAA, 1998, pp. 437-444.

[13] H. Lee and H. Bang, "Optimal steering laws for variable speed control moment gyros," presented at the AIAA GN\&C Conf., San Francisco, CA, Aug. 2005.

[14] H. Lee and H. Bang, "Singularity avoidance of variable speed control moment gyros by optimization approach," J. Astronautical Sci., vol. 55 , no. 1 , pp. $67-84,2007$.

[15] H. Leeghim, H. Bang, and J.-O. Park, "Singularity avoidance of control moment gyros by one-step ahead singularity index," Acta Astronautica, vol. 64, no. 9-10, pp. 935-948, 2009.

[16] V. J. Lappas, W. H. Steyn, and C. Underwood, "Attitude control for small satellites using control moment gyros," Acta Astronautica, vol. 51, no. 1-9, pp. 101-111, 2002.

[17] S.-M. Lee and S.-W. Rhee, "Experiments of singularity avoidance steering control laws for redundant single-gimbal control moment gyros," presented at the Int. Conf. Control, Autom. Syst., Seoul, Korea, Oct. 2007.

[18] J.-W. Kim, R. Cristi, and B. N. Agrawal, "Attitude determination for NPS three-axis spacecraft simulator," presented at the AIAA/AAS Astrodynamics Specialist Conf. Exhibit, Providence, RI, 2004, AIAA21287.

[19] V. J. Lappas, W. H. Steyn, and C. Underwood, "Design and testing of a control moment gyroscope cluster for small satellites," J. Spacecraft Rockets, vol. 42, no. 2, pp. 729-739, 2005.

[20] D. Jung and P. Tsiotras, "An experimental comparison of CMG steering control laws," presented at the AIAA/AAS Astrodynamics Specialist Conf., Providence, RI, Aug. 2004.

[21] J. Ahmed, R. H. Miller, E. H. Koopman, and V. T. Coppola, "An actively controlled control moment gyro/gyropendulum testbed," in Proc. IEEE Int. Conf. Control Appl., Oct. 1997, pp. 250-252.

[22] K. A. Ford and C. D. Hall, "Flexible spacecraft reorientations using gimbaled momentum wheels," in Proc. Adv. Astronautical Sci., Astrodyn., San Diego, CA, 1997, vol. 97, pp. 1895-1914.

[23] B. Wie, "Singularity analysis and visualization for single-gimbal control moment gyro systems," J. Guid., Control, Dyn., vol. 27, no. 2, pp. 271-282, 2004.

[24] H. Yoon and P. Tsiotras, "Singularity analysis of variable-speed control moment gyros," J. Guid., Control, Dyn., vol. 27, no. 3, pp. 374-386, 2004.

[25] A. E. Bryson, Jr. and Y.-C. Ho, Applied Optimal Control. Washington, DC: Hemisphere, 1975.

[26] E. Kreyszig, Introductory Functional Analysis With Applications. New York: Wiley, 1989. 